\title{
Model-Based Approach to Investigate the Influences of Different Load States to the Vehicle Dynamics of Light Electric Vehicles
}

\author{
Harry Ott ${ }^{1}$, René Degen1, Mats Leijon ${ }^{2}$, Margot Ruschitzka1 \\ ${ }^{1}$ CAD CAM Center Cologne, Institute of Automotive Engineering Cologne (IFK), Faculty of Automotive Systems and Production, \\ Cologne University of Applied Science, Cologne, Germany \\ ${ }^{2}$ Division of Electricity, Department of Electrical Engineering, Uppsala University, Uppsala, Sweden \\ Email: *harry.ott@th.koeln.de
}

How to cite this paper: Ott, H., Degen, R., Leijon, M. and Ruschitzka, M. (2021) Model-Based Approach to Investigate the Influences of Different Load States to the Vehicle Dynamics of Light Electric Vehicles. Journal of Transportation Technologies, 11, 213-230.

https://doi.org/10.4236/jtts.2021.112014

Received: February 5, 2021

Accepted: April 3, 2021

Published: April 6, 2021

Copyright $\odot 2021$ by author(s) and Scientific Research Publishing Inc. This work is licensed under the Creative Commons Attribution International License (CC BY 4.0).

http://creativecommons.org/licenses/by/4.0/

\begin{abstract}
The need to find alternative urban mobility solutions for delivery and transport has led mobility companies to devote enormous resources for researchbased solutions to increase vehicle safety. This paper documents a virtual approach to investigate the influences of different load states to the vehicle dynamic of light electric vehicle. A model basing on a three-dimensional multibody system was used, which consists of five bodies. By applying methods of multibody modelling the generalized equations of motion were generated. To include the behavior within the contact point between road and vehicle a simplified tire models was added. The implementation of the equations allowed a first validation of the model via simulations. In a final modeling step the simulation results were interpreted in respect of plausibility. Afterwards, the model was simulated numerically to investigate different load states of the vehicle, by applying constant steering stimuli and variable velocities. In sum, the investigated model approach is useful to identify safety relevant parameters and shows the effects of load states to the vehicle dynamics. Furthermore, it behaves plausibly regarding general vehicle dynamics. These results prove the general usability of the model for the development controllers and estimators in driver assistances systems.
\end{abstract}

\section{Keywords}

Vehicle Dynamics, Multibody System, Tricycle, Rigid Model, Numerical Simulation

\section{Introduction}

In the recent past, electric light vehicles have regained importance as urban mobili- 
ty solutions, what is confirmed by increasing unit sales in Europe [1] [2]. This kind of vehicle is especially interesting for the lightweight transportation sector because of the small amounts of space needed for parking and maneuvering operations.

This causes the need of intelligent, traffic-safety increasing systems to improve urban traffic. By just increasing mechanical stability and driving force, higher loads could be transported, but without respect to the vehicle safety and the vehicle dynamics.

Model based approaches offer a cost-effective opportunity for the development of overall light electric vehicle solutions. Such an approach must ensure a high degree of flexibility.

For instance, the positions, in which physical quantities are calculated within the model, should be adaptable to variable positions of sensors within the real prototype. This is due to measured quantities like the centrifugal acceleration varies by changing the sensors position within a vehicle. The modelling approach presented below allows adding any number of coordinate systems. Therefore, physical quantities acting in the points of origin of a system dedicated to a specific sensor can be calculated for an appropriate comparison with measurement data [3] [4]. Because the field of research engaged in dedicated modelling is still narrow it is our aim to broaden the foundation for further model-based developments of specific assistance systems.

The main objectives of this study are the development of a vehicle model, which enables quantity variation. Additionally, the specific goals are:

- A mathematical model for the description of the dynamics of a representative light electric vehicle.

- The model-verification by means of selected investigation maneuvers.

- The analysis of additional masses to the vehicle behavior.

\section{Conceptual Reviews/State of Research}

\subsection{Configuration of Three-Lane Vehicle System}

Three-track small vehicles can have a wide variety of configurations. They can have two wheels in the front and one wheel in the rear (2F1R) or, conversely, one wheel in the front and two wheels in the rear (1F2R). In the case of threetrack vehicles for cargo transportation, the area of an additional load can be located in the front area or in the rear area. The latter is the most common. Also, in many three-lane vehicles there is a tendency to lean in the direction of the curve, similar to a bicycle. This paper is not focused on such three-lane vehicles. However, Figure 1 shows the topology of a three-lane vehicle on which this paper focuses.

\subsection{Review of Empirical Literature}

The literature review below is divided into two main areas. On the one hand, an overview of the modeling approaches and the analysis focus of three-lane vehicles is given. On the other hand, a more general overview of modeling approaches for the investigation of the load states to the driving behavior is given. 


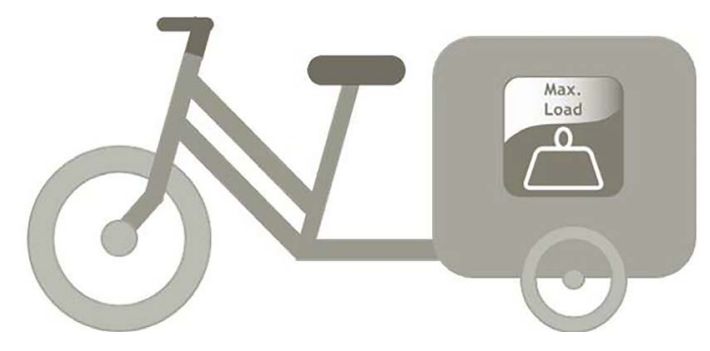

Figure 1. Topology of the three-lane reference vehicle.

The objective of modeling the dynamics of three-lane vehicles has a wide field of application with different focal points of investigation. In [5] a simulation model is described with the aim of investigating the structure vibrations with respect to the ride comfort. The simulation model based on the method of multi-body modeling. Due to the investigation aspect, however, the model has only one longitudinal degree of freedom. For this reason, this model variant cannot be used for an investigation of lateral movements. The same applies to [6]. Here, too, only the longitudinal degree of freedom is taken into account, with the focus of investigation on the electric drive train in terms of efficiency. The editors of the paper [7] focuses within his investigation to the influence of geometric parameters on the stability of various three-lane vehicle topologies. The roll over threshold is used for evaluation. The basis of the investigation is a highly simplified model that is based on static equations. Based on the lower stability of threelane vehicles, some three-wheeled vehicles have a more complex mechanical tilt mechanism. The investigation of the stability on such vehicles is carried out in [8] and [9], among others. Here, models according to the Newton-Euler method are applied. Modeling focus is the representation of the inclination during cornering. The overarching goal of these works is to develop tilt controllers. A first general modeling approach to describe longitudinal and lateral dynamics for three-lane vehicles is provided by [10]. Here, a model for a $2 \mathrm{~F} 1 \mathrm{R}$ vehicle is developed from several simplified models using Newton's and Euler's equations. The tire model is represented by maps that generate different longitudinal and lateral forces depending on different ground conditions. Finally, some simulations are performed for plausibility investigations, focusing on different ground conditions. The model offers the possibility to shift the center of mass arbitrarily, which is generally not discussed further. A similar model is built in [11]. Based on the same modeling method, a simulation model is derived and developed here for the 1F2R vehicle. The objective of the development of this model was on the one hand the investigation of the tilting stability and on the other hand the model was used for the development of a torque controller for an electric motor. In relation to four-wheeled vehicles, in which there are model variants with a wide variety of complexities and levels of detail, the amount of modeling approaches or simulation models is relatively small, despite the potential to participate in the development of driver assistance systems. To complete the literature review with respect to the objective of the paper, some papers dealing with the influence of 
the loading condition will be presented below.

In [12], the influence of the load on the braking behavior of light transport vehicles is investigated. Methodically, simple static equations and several measurement experiments are used. The investigation of the influence of the height changes of the entire center of gravity, which can result from additional loading, is presented in [13] and [14]. Here again simple basic static equations are discussed. Due to the influence of the loading condition on the driving behavior, [15] and [16] already deal with methods to estimate the changed vehicle mass or center of gravity height. To summarize, the model in this paper differs from the other models in the way that this model has a high flexibility with the possibility of analyzing the effect of different loads on the behavior of different drive systems.

\section{Research Methodology}

This chapter describes a stepwise modelling procedure in which the successive steps cover different fields of mechanics. Foundation for the modelling is a physical model scheme as simplified description of the tricycles mechanics. It is designed as multibody system with discrete components for springs and dampers, which represent their ideal physical behavior [3]. Each body of the system has its own coordinate system. Based on this scheme the multibody systems kinematics of positions are formulated. Thereby, the absolute and relative positions of all bodies are described using vectors and rotational matrices. The kinematics of velocity and acceleration are successively calculated by deviation. Meanwhile, Jacobian matrices are generated, which map generalized quantities of the multibody system onto actual quantities of all bodies [3] [4]. For this kind of description, tools like coordinate transformations and formulas of relative kinematics are used [4] [17]. After the systems kinematics are known a description of the kinetics is derived. A free-body system is generated by the methods of sections as foundation for the kinetic description [17]. Hereinafter, the word "forces" is used summing up the word "forces" and "torques" as kinetic quantities. The free-body system contains all inner and outer forces per body as well as all constrained forces. Due to the known kinematic behavior, those forces can be expressed as function of kinematic quantities. For instance, a damper force depends on relative velocities of bodies connected by the damper. Afterwards, these forces are summed up in addition of inertial, centrifugal and coriolis forces producing newton and euler equations per body [3]. Merging the newton and euler equations of all bodies within a vector generates the newton-euler equation of the multibody system [3]. In ordinary multi-body systems with holonomic constraints the Newton-Euler-Equation can be transformed to the generalized equations of motion of the Multi-body system using jacobian matrices. The structure of the generalized equations of motion is comparable to the structure of Lagrange's equations of the first kind [4]. They contain the degrees of freedom of the multibody system and their derivatives as variables [3]. To display 
the systems behavior, the generalized equations of motion can be solved numerically within an appropriate software environment.

\subsection{Model Introduction}

Foundation of the modelling procedure is an abstracted representation of the vehicle shown in Figure 2. It visualizes a multibody system with several characteristics defined below. The multibody system consists of five bodies. Each wheel is represented by one body. The bodies of the frame and the loading are represented by one mass point each. The indices $\mathrm{m}$ and $\mathrm{J}$ symbolize the mass and the mass moment of inertia per body. Each body includes a Cartesian coordinate system in its center of gravity. Furthermore, an inertial coordinate system is positioned at any place in space.

In this case the term "position" includes the term "orientation" as well. Besides these coordinate systems, several auxiliary coordinate systems are used. Subsequent to these basic specifications, the mechanical degrees of freedom are defined, which are marked by violet arrows and are listed in Table 1 . The sense of rotation for the rotary degrees of freedom is clockwise in direction of the double-arrows.

To ensure clarity, the discrete components mentioned in the previous chapter and all input variables are shown in Figure 3. Several assumptions are made to generate this abstract model of the tricycle. For instance, the geometrical parameters are neglect. The Rolling motion refers to an artificial rolling axis, which is located at road level. The pitching motion is neglected due to a focus on lateral dynamics. The masses of the wheels are assumed to contain the masses of motor, braking system and tire components. The frame mass sums up the masses of all vehicle components fixed to the frame. Furthermore, the frame and the wheels are assumed to be rigid. Moreover, the model is valid only for continuous roadcontact. A loss of contact can be modelled additionally.

\subsection{Kinematics}

In this section the description of the models kinematics is given. It consists of the kinematics of position, the kinematics of velocity and the kinematics of acceleration. These three areas of kinematics are deduced successively. They are exemplified by means of the vehicle-fixed coordinate system V. This is because the matrices of other bodies reach impractical large sizes and the mathematical procedures are similar.

In the first instance the kinematics of position are described. For that purpose absolute and relative positions vector and rotational matrices are defined for each body respectively coordinate system, which represent functions of the degrees of freedom. From a mathematical point of view, the degrees of freedom represent a set of generalized coordinates, which are now summarized in the following vector of the generalized coordinates [17].

$$
\underline{q}=\left[\begin{array}{lllllllll}
X_{V} & Y_{V} & \Psi & Z_{\text {body }} & \delta & \varphi & \gamma_{F} & \gamma_{R L} & \gamma_{R R}
\end{array}\right]^{\mathrm{T}}
$$




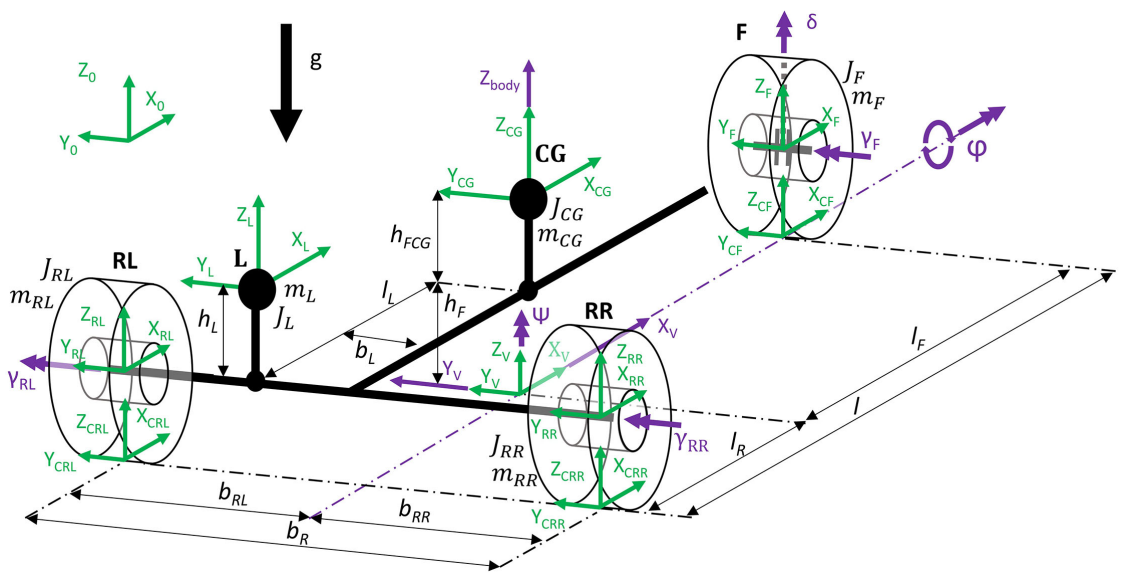

Figure 2. Representation of all degrees of freedom and coordinate systems of the vehicle.

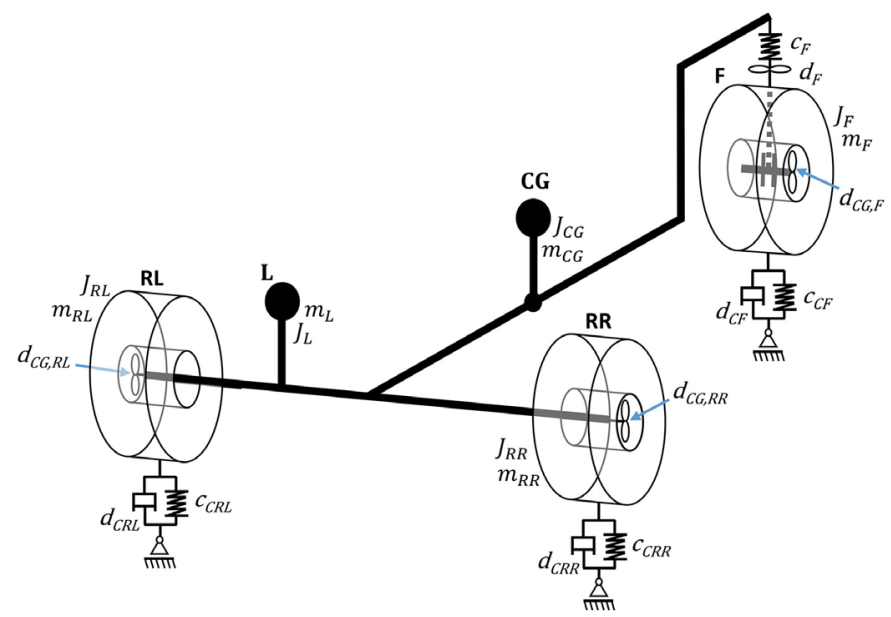

Figure 3. Discrete components and input parameters of the vehicle.

Table 1. Degrees of Freedom of the multibody system.

\begin{tabular}{cc}
\hline Symbol & Degree of freedom \\
\hline$\Psi$ & yaw angle of the frame \\
$\delta$ & steering angle of the front tire \\
$\phi$ & rolling angle of the frame \\
$\theta$ & pitching angle of the frame \\
$\gamma_{R R}, \gamma_{R L}, \gamma_{F}$ & rotation angle per tire \\
$X_{V}, Y_{V}$ & longitudinal and lateral translation of the vehicle \\
$Z_{b o d y}$ & vertical translation of frame body CG \\
\hline
\end{tabular}

The absolute position vector ${ }^{(0)} \underline{r}_{0, V}{ }^{1}$ and the relative rotational matrix ${ }^{(0, V)} \underline{S}^{2}$ as mentioned below.

${ }^{1}$ Notation ${ }^{(A)} \underline{r}_{B, C}$ : vector $r$ from the point of origin of coordinate system A to the point of origin of coordinate system $\mathrm{C}$ describe in the coordinates of system BA.

${ }^{2}$ Notation ${ }^{(A, B)} \underline{S}$ : rotational matrix transforming coordinates of system B to coordinates of system A. 


$$
\begin{gathered}
{ }^{(0)} \underline{r}_{0, V}=\left[\begin{array}{lll}
X_{V} & Y_{V} & 0
\end{array}\right]^{\mathrm{T}} \\
{ }^{(0, V)} \underline{S}=\left[\begin{array}{ccc}
\cos (\Psi) & -\sin (\Psi) & 0 \\
\sin (\Psi) & \cos (\Psi) & 0 \\
0 & 0 & 1
\end{array}\right]
\end{gathered}
$$

Basing on this description of the kinematics of position the modelling process continues by introducing the kinematics of velocity [4]. Therefore, the vector of the generalized velocities is introduced below.

$$
\underline{\dot{q}}=\left[\begin{array}{lllllllll}
\dot{X}_{V} & \dot{Y}_{V} & \dot{\Psi} & \dot{Z}_{\text {body }} & \dot{\delta} & \dot{\varphi} & \dot{\gamma}_{F} & \dot{\gamma}_{R L} & \dot{\gamma}_{R R}
\end{array}\right]^{\mathrm{T}}
$$

For generating the vectors of the translational and angular velocity, the positions vector and rotational matrices are derived and combined. Furthermore, the Jacobian matrices are created.

$$
\begin{gathered}
{ }^{(0)} \underline{v}_{0, V}=\frac{\mathrm{d}}{\mathrm{d} t}{ }^{(0)} \underline{r}_{0, V}=\left[\begin{array}{lll}
\dot{X}_{V} & \dot{Y}_{V} & 0
\end{array}\right]^{\mathrm{T}} \\
{ }^{\left({ }^{0, V)}\right)} \underline{\dot{S}}=\frac{\mathrm{d}}{\mathrm{d} t}{ }^{(0, V)} \underline{S}=\left[\begin{array}{ccc}
-\dot{\Psi} \cdot \cos (\Psi) & -\dot{\Psi} \cdot \sin (\Psi) & 0 \\
\dot{\Psi} \cdot \sin (\Psi) & -\dot{\Psi} \cdot \cos (\Psi) & 0 \\
0 & 0 & 1
\end{array}\right]
\end{gathered}
$$

Referring to [3] a matrix is defined, which has the angular velocities as elements. It can be shown, that this matrix is a skew-symmetric tensor.

$$
\begin{aligned}
{ }^{(V)} \underline{\tilde{\Omega}}_{V} & =\left({ }^{(0, V)} \underline{\underline{S}}\right)^{-1} \cdot{ }^{(0, V)} \underline{\dot{S}}={ }^{(0, V)} \underline{\underline{S}} \cdot{ }^{(0, V)} \underline{\dot{S}} \\
& =\left[\begin{array}{ccc}
0 & -\Omega_{z} & \Omega_{y} \\
\Omega_{z} & 0 & -\Omega_{x} \\
-\Omega_{y} & \Omega_{x} & 1
\end{array}\right]=\left[\begin{array}{ccc}
0 & -\dot{\Psi} & 0 \\
\dot{\Psi} & 0 & 0 \\
0 & 0 & 1
\end{array}\right]
\end{aligned}
$$

The vector of angular velocities is portioned as follows.

$$
{ }^{(V)} \underline{\Omega}_{V}=\left[\begin{array}{lll}
0 & 0 & \dot{\Psi}
\end{array}\right]^{\mathrm{T}}
$$

To map generalized quantities on actual quantities the Jacobian matrices of translation $\underline{V}_{T V}$ and rotation $\underline{V}_{R V}$ are created. Examples for dedicated derivations and applications can be found in the literature [3] [18].

$$
\begin{gathered}
{ }^{(0)} \underline{V}_{0, V}={ }^{(0)} \underline{V}_{T V}(\underline{q}) \cdot \underline{q}=\left[\begin{array}{ccccccccc}
1 & 0 & 0 & 0 & 0 & 0 & 0 & 0 & 0 \\
0 & 1 & 0 & 0 & 0 & 0 & 0 & 0 & 0 \\
0 & 0 & 0 & 0 & 0 & 0 & 0 & 0 & 0
\end{array}\right] \\
{ }^{(V)} \underline{\Omega}_{V}={ }^{(V)} \underline{V}_{R V}(\underline{q}) \cdot \underline{q}=\left[\begin{array}{lllllllll}
0 & 0 & 0 & 0 & 0 & 0 & 0 & 0 & 0 \\
0 & 0 & 0 & 0 & 0 & 0 & 0 & 0 & 0 \\
0 & 0 & 1 & 0 & 0 & 0 & 0 & 0 & 0
\end{array}\right]
\end{gathered}
$$

As preparation for a later application, all Jacobian matrices of the bodies are merged within a matrix. In the literature, this matrix is called global Jacobian matrix [3]. 


$$
\underline{H}(\underline{q})=\left[\begin{array}{c}
{ }^{(0)} \underline{H}_{T, C G}(\underline{q}) \\
\vdots \\
{ }^{(0)} \underline{H}_{T, L}(\underline{q}) \\
{ }^{(C G)} \underline{H}_{R, C G}(\underline{q}) \\
\vdots \\
{ }^{(L)} \underline{H}_{R, L}(\underline{q})
\end{array}\right]
$$

Based on the kinematics of position and velocity, it is now possible to derive the kinematics of acceleration for all components of the multibody system. Therefore, the vector of the generalized accelerations is defined.

$$
\underline{\ddot{q}}=\left[\begin{array}{lllllllll}
\ddot{X}_{V} & \ddot{Y}_{V} & \ddot{\Psi} & \ddot{Z}_{\text {body }} & \ddot{\delta} & \ddot{\varphi} & \ddot{\gamma}_{F} & \ddot{\gamma}_{R L} & \ddot{\gamma}_{R R}
\end{array}\right]^{\mathrm{T}}
$$

The acceleration quantities are not described in more detail within this paper, because they arise by simple derivation of the quantities of position and velocity. The notation meets the one used for the velocities.

\subsection{Kinetics}

Based on a fund definition of the kinematics, it is now possible to proceed with the description of the kinetics. The physical model scheme of the previous chapter is adapted for this purpose as shown in Figure 4. It now shows all outer forces affecting the system.

The wheels are driven by a drive respectively brake torques per wheel. The front wheel is controlled by a torque, which adjusts the steering angle $\delta$. Furthermore, driving resistance forces as the air resistance $\underline{F}_{A I R}$ and the tire forces $\underline{F}_{C}$ are considered. These forces are calculated in separate models. The force $\underline{F}_{A I R}$ is assumed to have a longitudinal component only and to generate no torques. It sums up the air resistance forces for the whole vehicle. In Addition to the model scheme shown above, a free-body system is designed per body. The figure for the frame is shown as example in Figure 5. It contains the constraint forces (blue) symbolizing the connections between the bodies. In this context they are comparable to reaction forces. Furthermore, the weight forces and damping torques between the frame and the wheels are shown in this figure.

The components of the same force are merged in vectors to allow compact notations. Using this notation, the newton and euler vector equations can be formed. All forces affecting the multibody system are summarized in this equations [3].

$$
\begin{gathered}
m_{C G} \cdot{ }^{(0)} \underline{a}_{C G}=\sum^{(0, C G)} \underline{S} \cdot{ }^{(C G)} \underline{F} \\
{ }^{(C G)} \underline{D}_{C G}+{ }^{(C G)} \underline{\Omega}_{C G} \times{ }^{(C G)} \underline{D}_{C G}=\sum^{(C G)} \underline{M}
\end{gathered}
$$

With the angular momentum as ${ }^{(C G)} \underline{D}_{C G}={ }^{(C G)} \underline{J}_{C G} \cdot{ }^{(C G)} \underline{\Omega}_{C G}$

After this procedure is applied to all bodies the resulting equations are merged within a vector, which contains the newton-euler-equations $\underline{E}$ of the whole multibody system. 


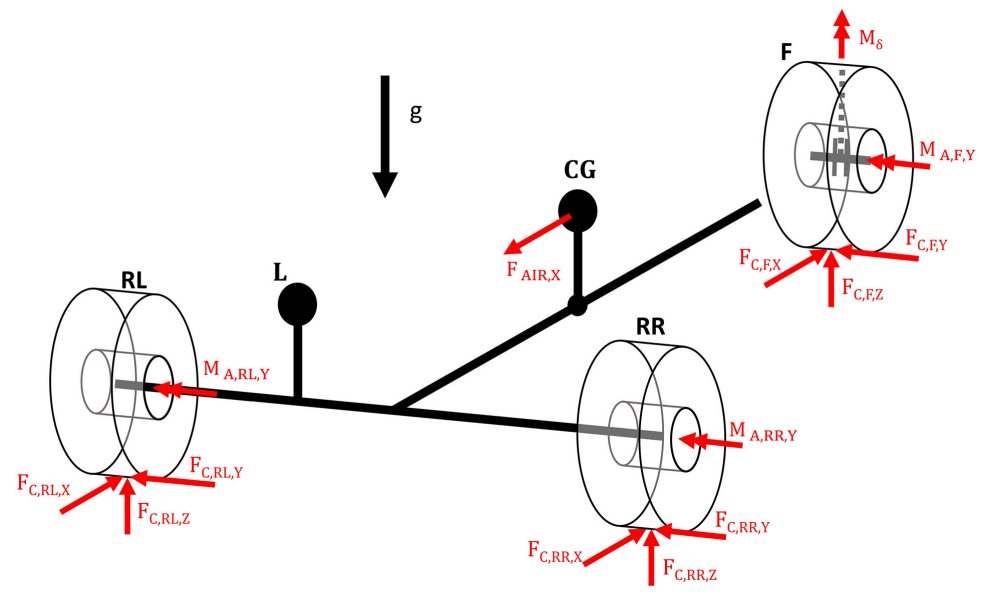

Figure 4. Impressed forces and moments on the vehicle.

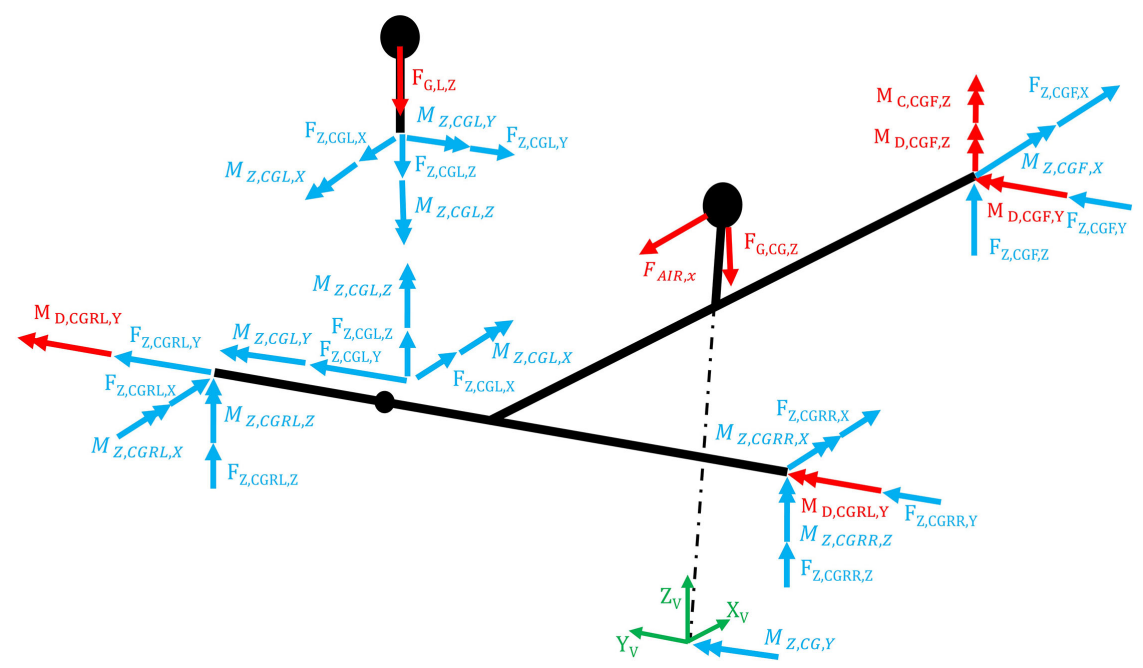

Figure 5. Free-body image of the frame with respective forces and moments.

$$
\underline{E}=\left[\begin{array}{c}
m_{C G} \cdot{ }^{\left({ }^{0}\right)} \underline{a}_{C G}-\sum^{\left({ }^{(0, C G)}\right.} \underline{S} \cdot{ }^{(C G)} \underline{F} \\
\vdots \\
m_{L} \cdot{ }^{\left({ }^{(0)}\right.} \underline{a}_{L}-\sum^{\left({ }^{(0, L)}\right.} \underline{S} \cdot{ }^{(L)} \underline{F} \\
{ }^{(C G)} \underline{D}_{C G}+{ }^{(C G)} \underline{\Omega}_{C G} \times{ }^{(C G)} \underline{D}_{C G}-\sum^{(C G)} \underline{M} \\
\vdots \\
{ }^{(L)} \underline{D}_{L}+{ }^{(L)} \underline{\Omega}_{L} \times{ }^{(L)} \underline{D}_{L}-\sum^{(L)} \underline{M}
\end{array}\right]=\left[\begin{array}{c}
0 \\
\vdots \\
0 \\
0 \\
\vdots \\
0
\end{array}\right]
$$

Multiplying this expression from the left side with the inverted matrix $\underline{H}$ transforms all actual quantities within $\underline{E}$ to generalized quantities.

$$
\underline{H}^{-1}(\underline{q}) \cdot \underline{E}=\underline{0}
$$

The results of this operation are the vectorial equation of motion of the multibody system shown below [3].

$$
\underline{M}(\underline{q}, t) \cdot \underline{\ddot{q}}+\underline{h}(\underline{q}, \underline{\dot{q}}, t)-\underline{f}(\underline{q}, \underline{\dot{q}}, t)=0
$$


It consists of $\underline{M}$ as global mass matrix, $\underline{h}$ as vector of the generalized gyroscopical (centrifugal and coriolis forces) and $f$ as vector of the generalized applied forces [18].

\subsection{Air Resistance and Tire Model}

In addition to the vehicle model described above, a simple representation of the air resistance and the tire behavior is necessary to calculate the forces $\underline{F}_{A I R}$ and $\underline{F}_{C}$. The former is reduced to the drag resistance and is defined by the equation below [19] [20].

$$
\underline{F}_{C}=\frac{1}{2} \cdot \rho \cdot c_{w} \cdot A \cdot X_{V}^{2}
$$

Simplified models are used for the longitudinal and lateral tire forces. The lateral tire force is generated by using the proportional relation with the side slip angle, which is valid for small side slip angles of " 5 degrees or less" according to [20]. The latter is determined with respect to the ratio of lateral and longitudinal velocities of the wheels. The longitudinal component is defined using the proportional relation with the wheel load in which the proportionality factor respectively the friction coefficient is a function of the tire slip.

\section{Simulation and Analysis}

\subsection{Implementation}

For the purpose of verification, the model equations defined above are added to MATLAB/Simulink. The vectorial equation of motion of the multibody system, the air resistance and the tire model are separately implemented within a Simulink block diagram.

In the following Table 2, the parameters used for the simulation setting are listed.

\subsection{Test Scenario}

With the objective of highlighting the dependency of the vehicle dynamics on the loading, several experiments are performed. These experiments mainly consist of the stimulation of the driving vehicle with a pulsed steering angle at a particular instant of time and of a subsequent interpretation of the systems response. Thus, this process is similar to the established experiment of using steering angle steps to investigate the transient behavior of vehicles [20]. To investigate the load dependence, these procedures are performed for different driving speeds and loads. Controllers are used to generate the torques for the desired steering angle $\delta$ and driving speed. It must be pointed out that the start time of fifteen seconds is necessary to reach the desired driving speeds. A stop time of seventeen seconds and a total duration of the experiment of forty seconds are chosen because the seemed to be appropriate to visualize the results. The driving speeds include an exemplary value for walking speed $(5 \mathrm{~km} / \mathrm{h})$, a speed half as large as 
the in town speed limit in Germany $(25 \mathrm{~km} / \mathrm{h}$ ) and this speed limit itself (50 $\mathrm{km} / \mathrm{h}$ ) [21] [22]. To generate a sufficiently high number of curves illustrating the load dependence of the dynamical behavior, six different cargo loads are used. These loads are selected to fit the gross vehicle weight of $300 \mathrm{~kg}$ [23].

The most important experimental parameters are listed within Table 3.

\subsection{Model Parametrization}

As preparation for the simulations, a set of parameters for the model must be defined. The parameters shown in Table 4 are roughly estimate, but serve the purpose of a model verification satisfactorily.

Table 2. Simulation Parameters used in MATLAB/Simulink.

\begin{tabular}{cc}
\hline Simulation Parameters & Description/Notation \\
\hline Numerical Solver & ODE45 (Dormand-Prince-method) \\
Maximum step size $[\mathrm{s}]$ & 0.01 \\
Relative tolerance $[\mathrm{s}]$ & 0.001 \\
\hline
\end{tabular}

Table 3. Experiment Parameters used in MATLAB/Simulink.

\begin{tabular}{|c|c|c|c|c|c|c|}
\hline Experiment Parameter & \multicolumn{6}{|c|}{ Value } \\
\hline Amount of $\delta\left[^{\circ}\right]$ & \multicolumn{6}{|c|}{5} \\
\hline Start time of $\delta$-pulse [s] & \multicolumn{6}{|c|}{15} \\
\hline End time of $\delta$-pulse [s] & \multicolumn{6}{|c|}{17} \\
\hline Duration of Experiment [s] & \multicolumn{6}{|c|}{40} \\
\hline Driving speed $[\mathrm{km} / \mathrm{h}]$ & \multicolumn{2}{|c|}{5} & \multicolumn{2}{|c|}{25} & \multicolumn{2}{|c|}{50} \\
\hline Cargo load $m_{L}[\mathrm{~kg}]$ & 0 & 30 & 60 & 90 & 120 & 150 \\
\hline
\end{tabular}

Table 4. Model Parameters used in MATLAB/Simulink.

\begin{tabular}{cc}
\hline Model Parameter & Value \\
\hline$m_{C G}[\mathrm{~kg}]$ & 150 \\
$I[\mathrm{~m}]$ & 1.7 \\
$b_{R}[\mathrm{~m}]$ & 0.8 \\
$I_{R}[\mathrm{~m}]$ & 0.85 \\
$I_{F}[\mathrm{~m}]$ & 0.85 \\
$m_{L}[\mathrm{~kg}]$ & See Table 3 \\
$b_{L}[\mathrm{~m}]$ & 0 \\
$I_{L}[\mathrm{~m}]$ & 0.2 \\
\hline
\end{tabular}




\subsection{Simulation Results and Interpretation}

After describing the environment and the parameters, the model behavior is verified in a first step. Subsequently, the behavior is examined for parameter dependencies. The verification is done by comparing the model behavior to the actual behavior of a cornering vehicle with respect to a chain of causes and effects. The steering angle pulse causes an increasing side slip angle at the front wheel. Due to the linear tire model, a front lateral force $F_{\mathrm{C}, \mathrm{F}, \mathrm{Y}}$ is immediately generated in the direction of the steering angle. The lateral force generates a torque around the vertical axis, which results in an increasing yaw rate. Due to the yaw process, a slip angle is generated at both rear wheels, what results in lateral forces as well. Because of the orientation of these rear lateral forces $F_{\mathrm{C}, \mathrm{RR}, \mathrm{Y}}$ and $F_{\mathrm{C}, \mathrm{RL}, \mathrm{Y}}$ relating to the vehicle's vertical axis, these forces generate a torque balancing the torque caused by the front wheels lateral force. A state of equilibrium is reached in which the transient processes of all dynamic variables are completed. This is referred to as constant cornering. The model shows this behavior in all examined speed ranges, as the following figures show on the basis of the yaw rate curve [18] [20].

The transient behavior mentioned above is strongly related to the vehicles speed as visible in Figures 6-8. Besides the different steady state magnitudes of the yaw rate, the curves show increasing settling times for higher speeds. This indicates, that the model is reaching the limits of stable driving behavior. Raising the speed also results in increasing sensitivity concerning loads differences. The higher the load, the greater the yaw rate for a specific vehicle speed. This is reproduced by the different course travelled with increasing speed and increasing load at constant steering angle. This is visualized in Figures 9-11.

The transient behavior mentioned above is strongly related to the vehicles speed as visible in Figures 6-8. Besides the different steady state magnitudes of the yaw rate, the curves show increasing settling times for higher speeds as shown in other modelling studies [24] [25]. This indicates that the model is reaching the limits of stable driving behavior. Raising the speed also results in increasing sensitivity concerning loads differences. The higher the load, the greater the yaw rate for a specific vehicle speed. The reason for this is, that the center of gravity and therefore the line of action of the centrifugal forces travel backwards for an increasing load. This shown by the different course travelled with increasing speed and increasing load at the same steering angle.

Furthermore, the investigation of driving dynamic parameters provides more detailed information about the influences of different load states. Hereinafter, the slip angle is defined as used in this investigation. The sideslip angle is the angle between the vehicle's longitudinal axis and the speed vector that deviates during cornering. Furthermore, the sideslip angle is an indicator of the cornering stability. A sideslip angle close to the vehicle's longitudinal axis is an indication of stable cornering.

The behavior of the sideslip angle in Figures 12-14 shows insightful results. 
For example, the sideslip angle has a positive value at slow speeds regardless of the load. With a higher load, the sideslip angle shows a smaller value, which is rather negligible at slow speeds. At a speed of $25 \mathrm{~km} / \mathrm{h}$, the sideslip angle overshoots due to increasing load-states. Next, the sideslip angle has values close to zero without additional loads, which causes a more stable cornering behavior at $25 \mathrm{~km} / \mathrm{h}$. By increasing speed, the behavior changes, so that at $50 \mathrm{~km} / \mathrm{h}$ the maneuver is more stable around the curve without a load. The direction of the speed vector also changes with respect to the longitudinal axis, which is reflected by the driving behavior.

In sum, the thesis, that the model's dynamic is directly influenced by different load states is confirmed by these results. Therefore, the model behaves plausible.

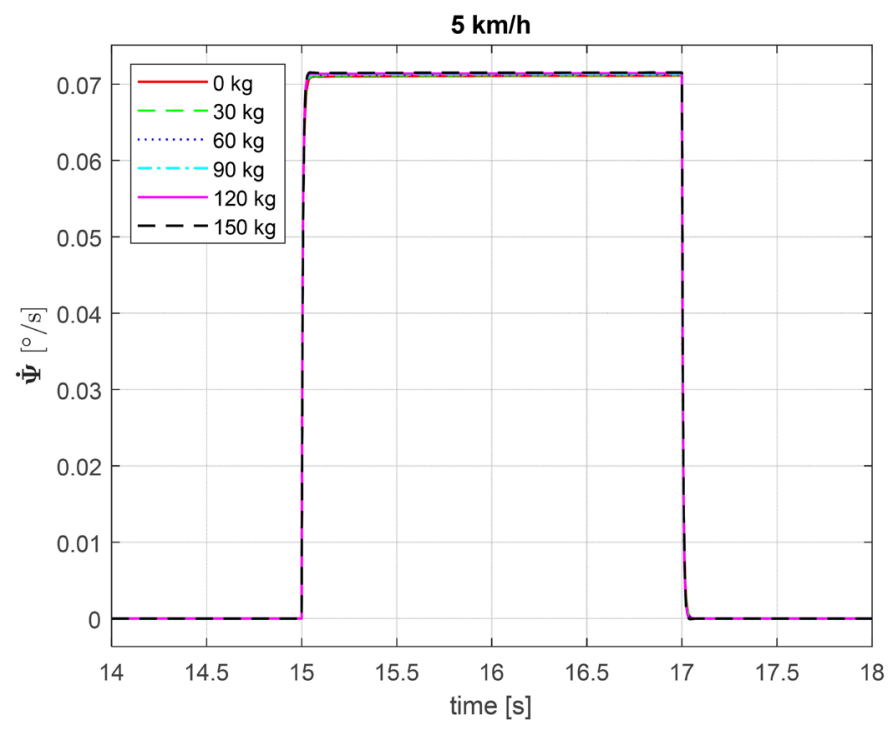

Figure 6. Transient response of yaw rate at a velocity of $5 \mathrm{~km} / \mathrm{h}$.

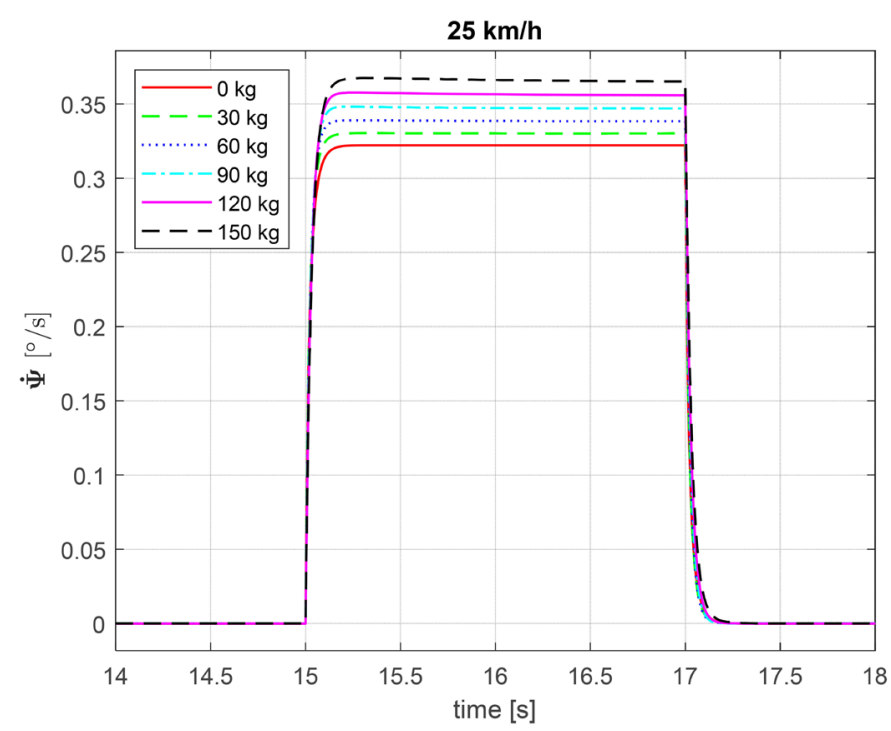

Figure 7. Transient response of yaw rate at a velocity of $25 \mathrm{~km} / \mathrm{h}$. 


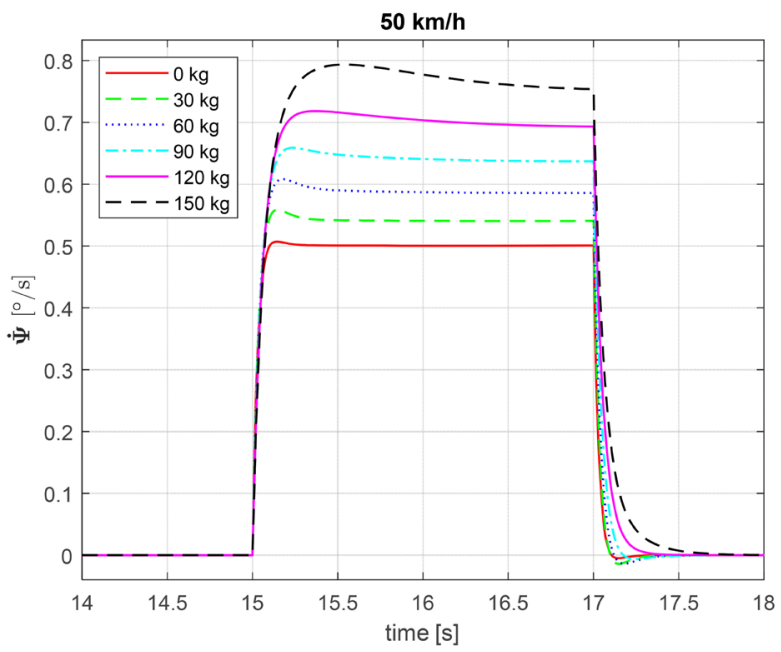

Figure 8. Transient response of yaw rate at a velocity of $50 \mathrm{~km} / \mathrm{h}$.

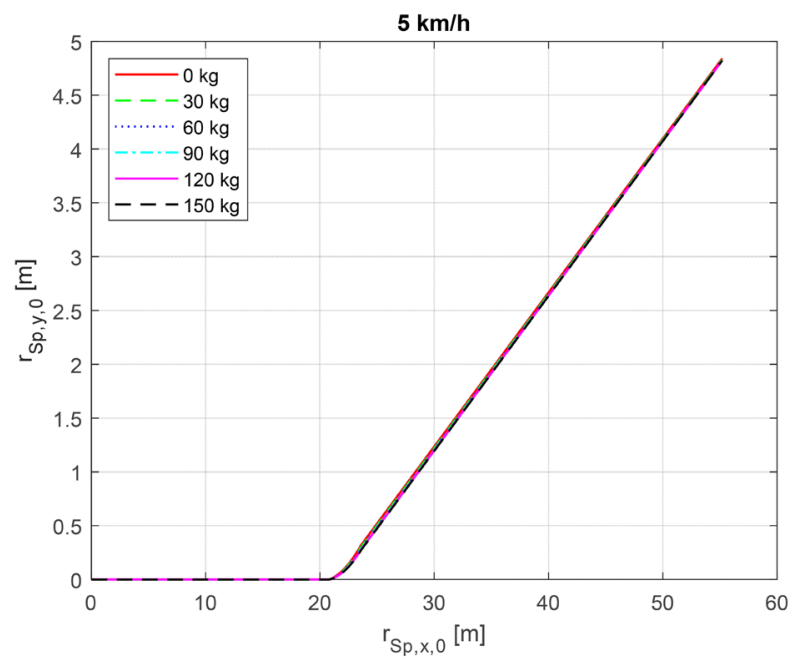

Figure 9. Driving course at a velocity of $5 \mathrm{~km} / \mathrm{h}$.

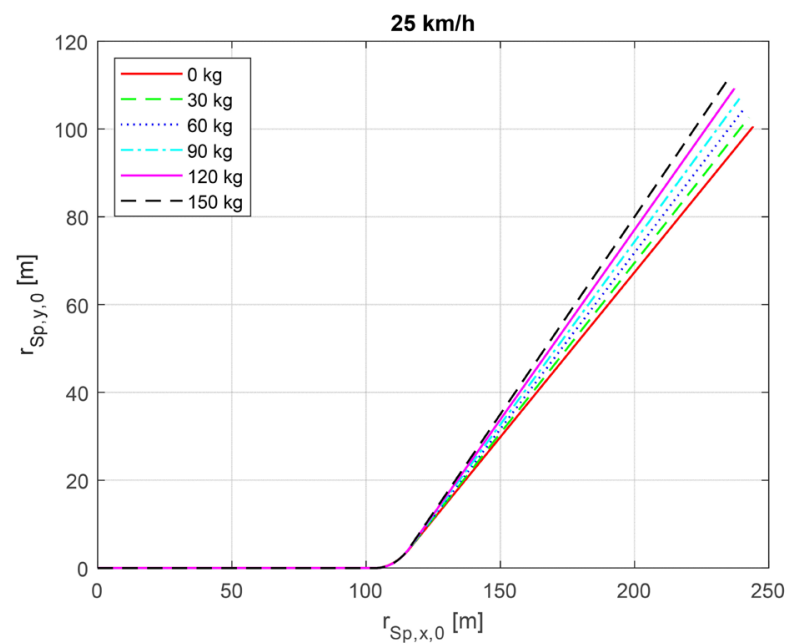

Figure 10. Driving course at a velocity of $25 \mathrm{~km} / \mathrm{h}$. 


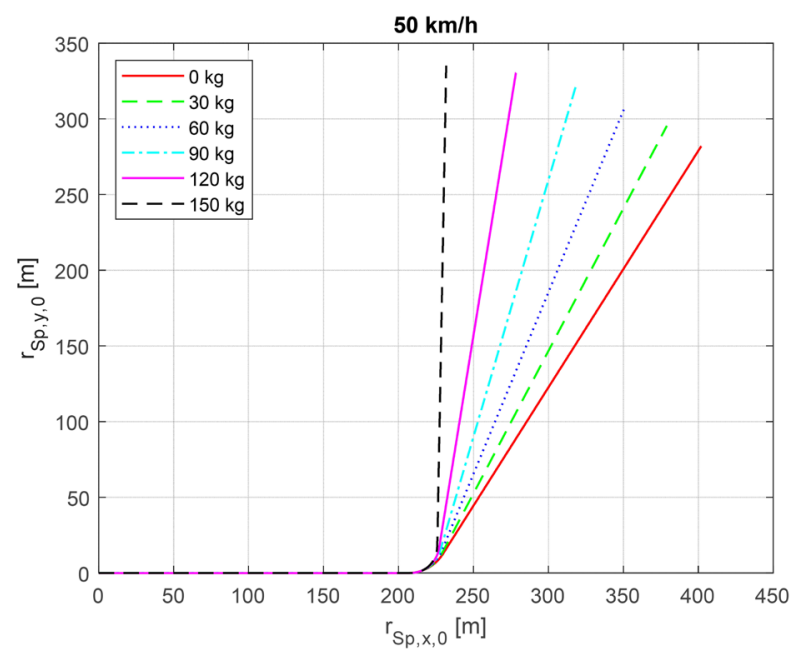

Figure 11. Driving course at a velocity of $50 \mathrm{~km} / \mathrm{h}$.

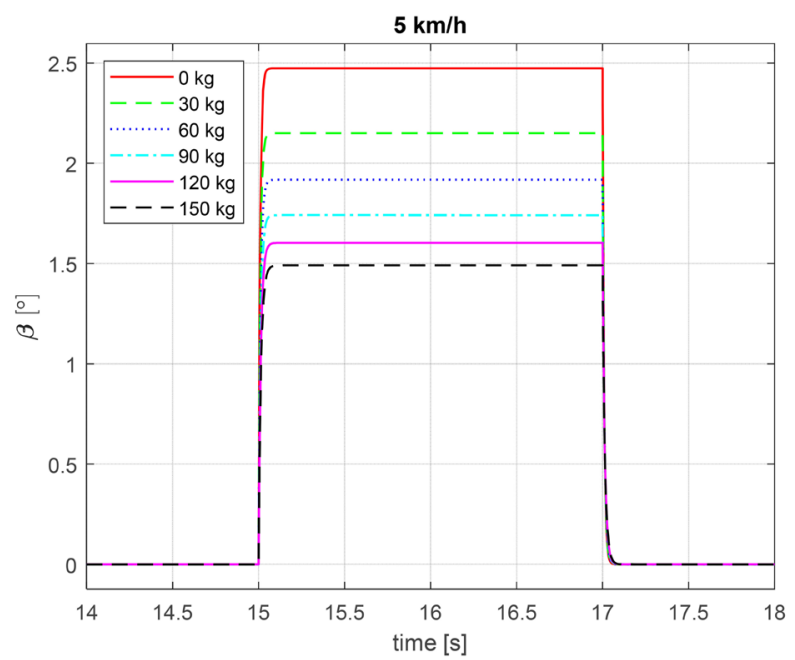

Figure 12. Transient response of side slip angle at a velocity of $5 \mathrm{~km} / \mathrm{h}$.

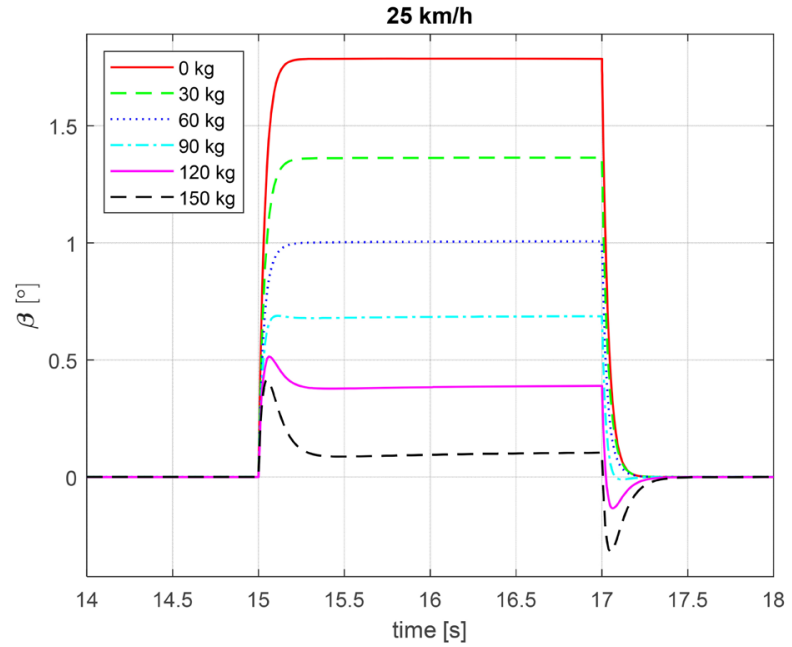

Figure 13. Transient response of side slip angle at a velocity of $25 \mathrm{~km} / \mathrm{h}$. 


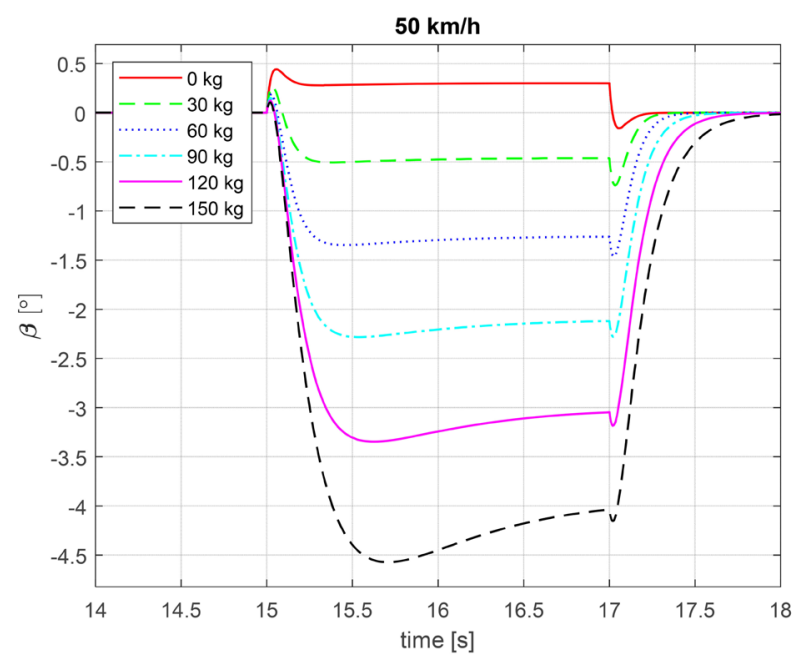

Figure 14. Transient response of side slip angle at a velocity of $50 \mathrm{~km} / \mathrm{h}$.

\section{Summary/Conclusion}

In this study, the development of a three-wheeled light electric vehicle is presented. Thereby, the main focus lays at the implementation of dynamical load states. The model could be verified by using steering stimuli at different driving speeds. Due to the possibility of large loads and its percentage of the total weight of the presented vehicle, there is the possibility of adding a load in the form of a rigid mass. In addition, its geometric position can be shifted in all axial directions. Next to the verification, a general simulation study is carried out in which the behavior of an additional loading mass at the vehicle is investigated. This was implemented by successively increasing the load sate at different driving speeds. It could be shown that from a speed of approx. $25 \mathrm{~km} / \mathrm{h}$, the load have a significant influence on the driving behavior of that specific vehicle.

In conclusion, the presented model can serve as a basis for the development of an electric differential or vehicle assistance systems. In following research activities, the model will first be validated with the help of defined scenarios and the measurement data obtained from them. For this purpose, a measuring unit is currently being built that can be attached to the vehicle and transmits the generated data to a development computer. Furthermore, the model of an electric drive train including a control system has to be connected to the presented model.

\section{Acknowledgements}

The Project is supported by the Ministry of Economic Affairs, Innovation, Digitization and Energy of North Rhine-Westphalia.

\section{Conflicts of Interest}

The authors declare no conflicts of interest regarding the publication of this paper. 


\section{References}

[1] Behrensen, S. (2020) Cargo Bike Boom in Europe. http://cyclelogistics.eu/sites/default/files/downloads/Press release Survey Market Size.pdf

[2] Llorca, C. and Moeckel, R. (2020) Study of Cargo Bikes for Parcel Deliveries under Different Supply, Demand and Spatial Conditions. 2020 Forum on Integrated and Sustainable Transportation Systems, Delft, 3-5 November 2020, 39-44. https://doi.org/10.1109/FISTS46898.2020.9264864

[3] Schiehlen, W. and Eberhard, P. (2014) Applied Dynamics. Springer, Cham. https://doi.org/10.1007/978-3-319-07335-4

[4] Pfeiffer, F. (2008) Mechanical System Dynamics. Springer-Verlag, Berlin, Heidelberg.

[5] Dizo, J. and Blatnicky, M. (2019) Evaluation of Vibration Properties of ThreeWheeled Vehicle in Terms of Comfort. Manufacturing Technology, 19, 197-203. https://doi.org/10.21062/ujep/269.2019/a/1213-2489/MT/19/2/197

[6] Sreejith R., Rajagopal, K.R. and Singh, B. (2016) Modelling and Analysis of PMBLDC Motor Based Three Wheeler EV for Closed Loop Optimum Operation. 2016 IEEE International Conference on Power Electronics, Drives and Energy Systems, Trivandrum, 14-17 December 2016, 1-5. https://doi.org/10.1109/PEDES.2016.7914520

[7] Sindha, J., Chakraborty, B. and Chakravarty, D. (2015) Rigid Body Modeling of Three Wheel Vehicle to Determine the Dynamic Stability-A Practical Approach. 2015 IEEE International Transportation Electrification Conference, Chennai, 27-29 August 2015, 1-8. https://doi.org/10.1109/ITEC-India.2015.7386889

[8] Furuichi, H., Huang, J., Matsuno, T. and Fukuda, T. (2012) Dynamic Model of Three Wheeled Narrow Tilting Vehicle and Corresponding Experiment Verification. 2012 IEEE/RSJ International Conference on Intelligent Robots and Systems, Vilamoura, 7-12 October 2012, 3728-3733. https://doi.org/10.1109/IROS.2012.6386033

[9] Sindha, J., Chakraborty, B. and Chakravarty, D. (2018) Automatic Stability Control of Three-Wheeler Vehicles-Recent Developments and Concerns towards a Sustainable Technology. Proceedings of the Institution of Mechanical Engineers, Part D. Journal of Automobile Engineering, 232, 418-434. https://doi.org/10.1177/0954407017701285

[10] Vasiljevic, G., Vrhovski, Z. and Bogdan, S. (2012) Dynamic Modeling and Simulation of a Three-Wheeled Electric Car. 2012 IEEE International Electric Vehicle Conference, Greenville, 4-8 March 2012, 1-8. https://doi.org/10.1109/IEVC.2012.6183186

[11] Ravikanth, G.S.G. and Sujatha, C. (2017) Dynamic Modeling and Simulation of a Three-Wheeled Hub Motor Vehicle. 2017 IEEE Transportation Electrification Conference, Pune, 13-15 December 2017, 1-5. https://doi.org/10.1109/ITEC-India.2017.8333849

[12] Skrucany, T., Vrabel, J. and Kazimir, P. (2019) The Influence of the Cargo Weight and Its Position on the Braking Characteristics of Light Commercial Vehicles, Open Engineering, 10, 154-165. https://doi.org/10.1515/eng-2020-0024

[13] Reński, A. (2015) Investigation of the Influence of the Centre of Gravity Position on the Course of Vehicle Rollover. 24th Enhanced Safety of Vehicles Conference, Gothenburg, 8-11 June 2015.

[14] Mokrickova, L. and Rievaj, V. (2001) Position of the Center of Gravity and Driveability of the Vehicle. Scientific Journal on Transport and Logistics, 42, 108-115.

[15] Chen, M., Yin, G., Zhang, N. and Chen, J. (2016) Joint Estimation of Center of 
Gravity Position and Mass for the Front and Rear Independently Driven Electric Vehicle with Payload in the Start Stage. 2016 35th Chinese Control Conference, Chengdu, 27-29 July 2016, 1932-1937. https://doi.org/10.1515/eng-2020-0024

[16] Deng, Z., Chu, D., Tian, F., He, Y., Wu, C. and Pei, X. (2017) Online Estimation for Vehicle Center of Gravity Height Based on Unscented Kalman Filter. 2017 4th International Conference on Transportation Information and Safety, Banff, 8-10 August 2017, 33-36. https://doi.org/10.1109/ICTIS.2017.8047738

[17] Pfeiffer, F. and Schindler, T. (2015) Introduction to Dynamics. Springer-Verlag, Berlin, Heidelberg.

[18] Popp, K. and Schiehlen, W. (2010) Ground Vehicle Dynamics. Springer-Verlag Berlin Heidelberg. https://doi.org/10.1007/978-3-540-68553-1

[19] Gillespie, T. (1992) Fundamentals of Vehicle Dynamics. SAE International, Warrendale. https://doi.org/10.4271/R-114

[20] Jazar, N. (2018) Vehicle Dynamics: Theory and Application. 3rd Edition, SpringerVerlag, New York.

[21] Mohler, B.J., Thompson, W.B., Creem-Regehr, S.H., Pick Jr., H.L. and Warren Jr., W.H. (2007) Visual Flow Influences Gait Transition Speed and Preferred Walking Speed. Experimental Brain Research, 181, 221-228.

https://doi.org/10.1007/s00221-007-0917-0

[22] German Road Traffic Act, Section 3.1 (2020). https://www.gesetze-im-internet.de/stvo 2013/ 3.html

[23] Kruse, A. (2021) TRILINER ${ }^{\oplus}$ ST2426, ST2426e, ST26, ST26e. https://rytle.de/triliner/

[24] Parra, A., Cagigas, D., Zubizarreta, A., Rodríguez, A.J. and Prieto, P. (2019) Modelling and Validation of Full Vehicle Model Based on a Novel Multibody Formulation. IECON 2019-45th Annual Conference of the IEEE Industrial Electronics Society, Lisbon, 14-17 October 2019, 675-680. https://doi.org/10.1109/IECON.2019.8926854

[25] Setiawan, J.D., Safarudin, M. and Singh, A. (2009) Modeling, Simulation and Validation of 14 DOF Full Vehicle Model. International Conference on Instrumentation, Communication, Information Technology, and Biomedical Engineering 2009, Bandung, 23-25 November 2009, 1-6. https://doi.org/10.1109/ICICI-BME.2009.5417285 